\title{
Revolución y salud del niño en Cuba
}

\author{
Revolution and the child's health in Cuba
}

\section{María Cecilia Santana Espinosa}

Especialista de II Grado en Pediatría. Profesora Auxiliar. Escuela Nacional de Salud Pública. La Habana, Cuba.

\section{RESUMEN}

El estado actual de salud de niños y niñas en Cuba es resultante de la política llevada a cabo por el Gobierno Revolucionario a lo largo de los últimos 50 años. EI presente artículo tiene como objetivo identificar los principales eventos que en el ámbito social y en el sector de la salud produjeron cambios en la salud de niños y adolescentes desde el propio triunfo de la Revolución en 1959. La metodología aplicada incluyó revisión documental y criterios de algunos de sus protagonistas. Se concluye que la salud integral de este sensible grupo poblacional muestra positiva y sostenida evolución.

Palabras clave: Salud del niño, atención integral, equipo interdisciplinario, Cuba.

\begin{abstract}
The present state of the child's health in Cuba results from the revolutionary government's policies implemented throughout the last 50 years. This article was aimed at identifying the main events in the national social context and health sector that brought about changes in the health status of children and adolescents in Cuba after the triumph of the Revolution in 1959. The methodology included documentary review and the criteria expressed by some of the participants in these events. It was concluded that comprehensive health of this sensitive population group shows a positive and sustained progress.
\end{abstract}


Key words: Child's health, comprehensive care, interdisciplinary team, Cuba.

\section{ANTECEDENTES}

La salud de niños y niñas resulta a nivel mundial un capitulo abierto al debate por su vulnerabilidad ante eventos epidemiológicos, económicos, sociales o de los servicios que afectan el desarrollo de su potencial genético; el comportamiento de sus indicadores denuncia de forma silente que políticos y gobiernos subvaloran los determinantes sociales de desigualdad e iniquidad y no priorizan el tema en sus agendas de trabajo, lo que deja sin protección a este sensible grupo poblacional y compromete el futuro del mundo. ${ }^{1}$

Ejemplo de desigualdad resulta la brecha existente en el comportamiento de la mortalidad infantil hacia dentro de los países y entre países ricos y pobres, resultante de la tragedia que en el mundo viven los desposeídos, aunque esto no niega que en las últimas décadas se lograron avances en este problema de salud, aspecto que aparece recogido en el informe sobre el Estado Mundial de la Infancia 2008 de UNICEF, el mismo también reconoce la ocurrencia cada año de casi 10 millones de muertes en menores de un año y más de 27000 en niños preescolares que pudieron haberse evitado en su gran mayoría con medidas tan simples como la lactancia materna exclusiva, la inmunización, el tratamiento de los mosquitos con insecticida, la administración de suplementos de vitamina A y la implementación de un adecuado sistema de salud que priorice la atención de niños/as como grupo poblacional vulnerable. ${ }^{1-3}$

El descenso de la mortalidad infantil a escala global en la América Latina y el Caribe durante el periodo 1990-2006 (bajó la tasa de 55 a 27 fallecidos por mil nacidos vivos) en su análisis diferencial muestra lo alejados de este propósito que aún están los países con situación más critica en el área como es el caso Haití y Bolivia, el primero con tasa de mortalidad infantil superior a 70 por cada mil nacidos vivos. Estos resultados se fundamentan en las desigualdades e iniquidades para el acceso a servicios básicos como el abasto de agua potable, saneamiento, atención médica y nutrición adecuada lo que además de muertes causa daño al crecimiento y desarrollo infantil con sus características secuelas no sólo en lo físico sino también en lo intelectual, con impacto aún más elevado para el área rural y las comunidades marginales en cuyas poblaciones aparecen frecuentemente enfermedades infecciosas prevenibles por vacunas, tienen falta de educación, trabajo infantil y explotación sexual, esta última, epidemia social que afecta la salud mental, espiritual y moral de niños y adolescentes. ${ }^{1-4}$

Cuba no escapó a esa realidad regional por lo que al triunfo de la Revolución de 1959 encontró un panorama caracterizado por la incultura, el analfabetismo, déficit de escuelas para más de 600000 niños, sólo 6300 médicos y de ellos, la mayoría ejercían la medicina privada y el $65 \%$ radicaba en la capital a pesar de que el 34 $\%$ de su población residía en zonas rurales; elevado índice de insalubridad y como consecuencia una esperanza de vida inferior a 58 años. Sólo el $20 \%$ de las cubanas recibían atención institucional o calificada al parto, había ausencia de atención prenatal y a niños con problemas de salud, muy baja cobertura de vacunación, elevado índice de desnutrición, parasitismo, enfermedades trasmisibles 
causantes de tasas de mortalidad infantil superior a 60 fallecidos por cada 100 mil nacidos vivos, indicador este elaborado sobre registros no confiables. ${ }^{5,6}$

\section{LA REVOLUCIÓN EN LA SALUD}

En Cuba se organizó y garantizó de forma gratuita el proceso de atención a la salud de niños/as desde el propio triunfo de la Revolución en 1959, decisión posteriormente consagrada por la nueva Constitución de la República cuando definió la responsabilidad del Estado para con la salud ciudadana, articulando su ejecución desde la Ley de la Salud, que designa al sector de la salud responsable de la planificación, coordinación, ejecución y control de las acciones médicas de promoción, preventivas-curativas y de rehabilitación.

Para el caso especial de la salud de mujeres y niños el Ministerio de Salud Pública creó primero el Departamento y después la Dirección de Salud Materno Infantil, la que quedó responsabilizada para el diseño metodológico e implementación del Programa Nacional Materno Infantil que llegó a convertirse en paradigma del Sistema de Salud en Cuba, dada la sistematicidad mantenida en el cumplimiento de la estrategia asignada que incluyó actividades de promoción para un crecimiento y desarrollo normal, la prevención de riesgos y enfermedades, la atención especializada a niños y adolescentes portadores de enfermedades agudas, crónicas y emergentes y las acciones integradas de equipos interdisciplinarios e intersectoriales dirigidas a poblaciones pediátricas portadoras de discapacidades o afecciones neuropsicológicas. ${ }^{7,8}$

Los profundos cambios políticos, sociales y económicos que acontecieron en Cuba a partir del año 1959 y durante la década del 60 provocaron importante mejoría en las condiciones de vida de mujeres y niños favoreciendo la positiva transformación del cuadro de salud de niños y adolescentes. Dentro de los principales eventos vale citar la promulgación de Ley de Reforma Agraria (1959), la Ley de Reforma Educacional (1959), la creación de 19000 aulas escolares el $80 \%$ de ellas en áreas rurales, la realización en todo el país de la campaña de alfabetización (1961). ${ }^{6}$

En está década del 60 en el sector de la salud también se produjeron cambios que favorecieron al grupo población en estudio destacándose el inicio del servicio médico social (1960), la construcción de hospitales rurales, la introducción del programa masivo de vacunación el que contó con amplia participación social, el vasto programa de formación de personal médico y paramédico, el incremento de camas asistenciales dedicadas a la atención obstétrica y del niño, la creación en la provincia de Camagüey del primer hogar materno (1962), lo que favoreció la realización del parto institucional y la atención calificada al neonato, el fomento de la lactancia materna, la planificación de consultas de pediatría-puericultura, se iniciaron los Programas de Disminución de las Enfermedades Diarreicas Agudas (1967), de Atención Integral al Niño y a la Mujer (1968), quedaron constituidos los Grupos Nacionales y Provinciales de Pediatría y Ginecoobstetricia. ${ }^{9-12}$

En las décadas de los 70 s y 80 s continúa el mejoramiento de la salud integral de niños y adolescentes en Cuba estimulado y sostenido por el Programa de Reducción de la Mortalidad Infantil y las acciones dirigidas por el Departamento Materno Infantil del Ministerio de Salud Publica los que dieron paso en el año 1980 al Programa Nacional de Atención Materno I nfantil que consideró objetivos específicos de atención a la salud de la mujer (18 actividades), a niños y adolescentes (13 actividades) los que de alguna forma fueron incluidas en el programa de desarrollo de las especialidades de pediatría, ginecología y obstetricia, neonatología y cirugía 
pediátrica. El Programa para la Reducción del Bajo Peso al Nacer, el progresivo perfeccionamiento de la Atención Primaria de Salud, la incorporación del modelo de Atención del Medico y la Enfermera de la Familia, el perfeccionamiento y extensión a todas las provincias de las salas de terapias intensivas pediátricas, neonatales y perinatales, la creación del cardiocentro pediátrico "William Soler" y de la Red Nacional Cardiopediátrica, el perfeccionamiento de los servicios de neurocirugía y cirugía pediátrica, contribuyeron a la consolidación del mejoramiento de la salud de niños y adolescentes. ${ }^{13-19}$

El Programa de Atención Materno Infantil creado en la década del 90 resultado metodológico de la experiencia acumulada en el ámbito nacional y en acciones internacionales como el Plan de Acción de la Cumbre Mundial de la infancia, centró sus esfuerzos en el último periodo fundamentalmente en aspectos relacionados con la renovación tecnológica, dirigiendo los esfuerzos a la creación de laboratorios de nutrición parenteral y bancos de leche humana, la revitalización de las salas de terapia pediátricas y neonatales con tecnología de punta, la introducción de modernas técnicas en el campo de la genética prenatal, clínica y comunitaria, y acompañando esta línea de trabajo, un profundo proceso de perfeccionamiento de los recursos humanos. ${ }^{20}$

La continuidad en la atención al niño cubano se organizó según momentos del desarrollo biológico comenzando por la etapa prenatal caracterizado por el seguimiento especializado a cada gestante dado por la inclusión de la pesquisa de enfermedades genéticas, malformaciones congénitas, hepatitis $\mathrm{B}$ y del VIH/SIDA, la atención estomatológica a la embarazada como parte de prevención de la sepsis materna, la preparación psicofísica para el parto y a la pareja en materias de maternidad y paternidad responsable, actividades que favorecen la disminución del bajo peso al nacer y otros problemas de salud del neonato que afectan la sobrevivencia o la calidad de vida de los niños. La atención prenatal es complementada con la creación de los hogares maternos, los hospitales rurales en un primer momento y posteriormente hospitales obstétricos dotados de tecnologías que mejoraron el funcionamiento de los servicios de perinatología y las terapias neonatales. ${ }^{21}$

Las actividades programadas continúan en la etapa perinatal e infantil, ya que si importante resulta salvar la vida de un recién nacido dándole así su primera gran oportunidad, importante es garantizarle una adecuada calidad de vida y para ello fueron organizadas tempranamente las consultas de puericultura, actividad también conocida como "seguimiento al niño sano", que consideran en su funcionamiento actividades de promoción del crecimiento y desarrollo en correspondencia con el potencial genético individual, la orientación anticipada a los padres sobre los principales eventos por lo que transitará el niño en las próximas etapas, la prevención de problemas de salud evitables con especial atención al proceso de inmunización que para el país considera vacunas contra 13 enfermedades. Estas consultas se iniciaron en la década del 60 en los policlínicos integrales y se fueron perfeccionando progresivamente y extendiéndose desde la atención sólo al niño menor de un año en sus inicios, a la población preescolar en un segundo tiempo y ya desde la década del 80 abarca la atención al adolescente y la prenatal. ${ }^{9}$

Las consultas de puericultura no abordan sólo las necesidades de los niños para alcanzar un óptimo desarrollo social e intelectual, sino que su estrategia de protección y estimulación a estas esferas está presente en acciones que se realizan en los círculos infantiles en el caso de los niños con vinculo institucional y mediante el programa "Educa a Tu Hijo" para los no institucionalizados, este último, con acciones intersectoriales coordinadas por la Dirección de Educación Preescolar del Ministerio de Educación y está dirigido especialmente a niños de edades tempranas, con la asesoría técnica de un grupo interdisciplinario de trabajo del nivel nacional 
que tiene representación a nivel local y cuenta con soporte pedagógico (folletos) para uso de los promotores comunitarios. Por todo lo anterior, esta forma de atención se convierte en un método que de forma sencilla da respuesta a preguntas que con frecuencia se hacen los padres tales como ¿cómo es el niño?, ¿qué hacer para que el niño crezca y se desarrolle sano y feliz?, por lo que aporta herramientas para la estimulación del desarrollo afectivo, intelectual, motriz y del lenguaje, así como la formación de valores, hábitos higiénicos, cualidades morales, cuidados de su salud y la prevención de accidentes. ${ }^{22}$

En el servicio de neonatología, en la consulta de puericultura o en cualquier otra acción asistencial pueden detectarse signos o síntomas predictivos de alteración nueropsíquica o motora en un niño, lo que requiere de valoración especializada. Ante esta necesidad científicamente sustentada la Dirección Materno Infantil del Ministerio de Salud Publica estableció servicios especializados a nivel nacional dentro de los que merecen especial mención por sus exitosos resultados los de los hospitales pediátricos "William Soler" y "J uan Manuel Márquez" asumidos por equipos interdisciplinarios formados por pediatras, neonatólogos, enfermeras, psicólogos, neurólogos, neurofisiólogas, neurocirujanos, fisiatras, pedagogos, entre otros, que se encargan del diagnostico, tratamiento y rehabilitación de niños afectados, ocupándose además de la preparación de profesionales que desde diferentes especialidades conformaran los equipos provinciales que integran la red asistencial nacional en diferentes modalidades de atención. A esta gestión se sumó en la presente década del 2000 la creación de los grupos de atención temprana que con enfoque comunitario brindan sus servicios a estos niños desde instituciones del nivel local, propiciando así la preparación de padres, familias, médicos y maestros como parte del proceso de rehabilitación desde un algoritmo único que incluye un sistema de referencia y contra referencia para los casos que así lo necesiten. ${ }^{23}$

La salud del niño no puede verse sólo desde el crecimiento y desarrollo ya que con frecuencia el equilibrio salud-enfermedad se rompe a favor de esta última dando lugar entre otras situaciones a la aparición de enfermedades crónicas de la infancia las que han requerido de atención especial dada su connotación familiar y social. La atención de esta población demanda de elevada sensibilidad humana y tiene un alto costo financiero pero Cuba a pesar del riguroso bloqueo económico arrastrado por más de cuatro décadas asumió este problema y dotó a las instituciones pediátricas de moderna tecnología e intensificó la preparación de especialistas en centros de reconocido prestigio internacional, lo que ha permitido la conformación de equipos interdisciplinarios que dan seguimiento a problemas tan sensibles de salud como la insuficiencia renal o hepática crónica en niños que requieren de trasplante y rehabilitación, por lo que para garantizar su tratamiento se enfrentó la renovación física y tecnológica del servicio de nefrología del hospital pediátrico "Centro Habana" y la creación del servicio de hepatotrasplante del hospital pediátrico "William Soler" , centros de referencia nacional y coordinadores de la respectiva red nacional de diagnóstico y atención clínica en cada caso, lo que facilita su presencia en todas las provincias del país, acercando así la atención especializada al lugar de residencia del niño enfermo desde una profunda concepción ética y humanística. ${ }^{24}$

Un tema no menos importante y que ha requerido especial atención es el de los accidentes que sigue cobrando numerosas vidas de niños y adolescente a todo lo largo del país además de las lamentables secuelas en poblaciones hasta ese momento sanas, para atender este aspecto se creó un grupo interdisciplinario e intersectorial coordinado desde la Dirección Materno Infantil del Ministerio de Salud Publica por el respetado y querido pediatra cubano Francisco Valdés Lazo, el grupo ha diseñado las acciones metodológicas de prevención, planifica y ejecuta sistemáticamente actividades de capacitación y elabora materiales didácticos para uso individual, colectivo, a nivel local y por los medios de difusión masiva adecuándolos a las características de los diferentes entornos. ${ }^{25}$ 
La salud de los niños en ocasiones se quebranta totalmente y aparece el indicador negativo de mortalidad, el mayor numero de muertes pediátricas se registra en el subgrupo poblacional menor de un año, por lo que en cualquier estudio relacionado con el cuadro de salud del niño incluye este visible componente que habla del nivel de desarrollo social de un país. En Cuba el destacado pediatra ya fallecido Dr. Raúl Riverón Corteguera y el salubrista Dr. Pedro Azcuy Hernández, estudiaron el comportamiento de la mortalidad infantil durante el periodo 1959-1999, y encuentran una disminución de esta mortalidad en el periodo analizado (81,3\%), con positiva participación de sus tres componentes: la mortalidad neonatal precoz $(73,4 \%)$, la mortalidad neonatal tardía $(83,6 \%)$ y la posneonatal $(86,0 \%)$. Estos resultados estuvieron favorecidos por el incremento del parto institucional $(65,9$ $\%)$, con mayor distribución porcentual en las provincias centrales y occidentales; las principales causas de mortalidad se reportaron para las enteritis y otras enfermedades diarreicas, las infecciones respiratorias agudas, las afecciones perinatales en general y las meningitis aunque todas con disminución de su indicador; la tasa de mortalidad por bajo peso al nacer descendió en el 36,9 \%, la mortalidad perinatal se redujo el $67,2 \%$, los nacidos vivos disminuyeron el $21,1 \%$ y la supervivencia infantil a los 5 años aumentó el 3,8 \%. I gual comportamiento se ha mantenido para el periodo 2000-2007 lo que permitió alcanzar en los dos últimos años 2006-2007 los mejores indicadores de la historia con tasa de mortalidad infantil en ambos años de 5,3 fallecidos por cada mil nacidos vivos e índice de bajo peso al nacer de 5,4 \% sólo comparables con países desarrollados. ${ }^{26,27}$

Dos eventos en el área de las investigaciones sociales sirvieron de base para las principales estrategias desarrolladas desde el Programa Materno Infantil para beneficiar la salud integral de niños y adolescentes, el primero fue el estudio perinatal realizado completamente en Cuba con la contribución del Fondo de Naciones Unidad para Actividades en Materia de Población (FNUAP) que facilitó los servicios como expertos del Prof. Neville Butler y del estadístico Harvey Goldtein de Bristol y Oxford, respectivamente, involucró a todos los trabajadores del Ministerio de Salud Pública, otros sectores de la economía y contó con la entusiasta colaboración de las organizaciones de masas: Federación de Mujeres Cubanas y la Asociación Nacional de Agricultores Pequeños, lo que posibilitó que fueran encuestados el 99,1 \% de los nacimientos institucionales y extrainstitucionales, ocurridos en todo el territorio nacional en el período estudiado.

Esta investigación recogió datos sobre el total de nacimientos (nacidos vivos y muertes fetales) que ocurrieron en el país del 1ro. al 7 de marzo de 1973, se continuaron estudiando los nacimientos con peso inferior a $2500 \mathrm{~g}$ del 8 al 31 de marzo y la totalidad de las muertes perinatales que ocurrieron durante los meses de marzo, abril, y mayo de ese año. Se llenaron 4485 cuestionarios (de ellos 45 correspondieron a embarazos múltiples) los datos obtenidos sirvieron para conocer a nivel nacional, aspectos de la atención prenatal, de los cuidados intraparto, de las características maternas y de la atención al recién nacido. ${ }^{28}$

La investigación sobre Crecimiento Desarrollo, coordinada desde el Instituto de la Infancia por el prestigioso pediatra y salubrista Dr. José J ordán, estudia una muestra representativa de la población comprendida entre 0 y 19 años para evaluar no sólo el estado nutricional de la población seleccionada en un momento determinado, sino para tomarla como base para la determinación de la tendencia secular de los cubanos. ${ }^{29}$

Ya en la década del 90 impactaron en la salud de los niños cubanos dos estudios de extensión nacional y amplia participación intersectorial y social: el Diagnostico Nacional de Discapacidades y el Diagnostico del Estado Nutricional de los Niños Cubanos, ambos visualizaron desigualdades e iniquidades que aunque en bajo 
rango aún están presentes en el país. Estas investigaciones fueron realizadas por indicación del más alto nivel del Estado y a sus resultados se les prestó especial atención, designándose al sector de la salud para la coordinación de las necesidades de tratamiento diferenciado a cada caso con la fiscalización de los gobiernos locales. Costosas han resultado las inversiones necesarias para abordar los problemas detectados, tal es el caso de la sordo ceguera o la baja audición, los que además requieren de compleja intervención quirúrgica que corresponden a la modalidad de implantología y de rigurosa rehabilitación, de igual forma, demandaron modernización tecnológica programas ya existentes como los de atención a niños portadores de fibrosis quística del páncreas, enfermedad celiaca, xerodermia pigmentosa, entre otras afecciones. ${ }^{30}$ (Estudio del estado nutricional de los niños cubanos. 2003).

Finalmente se puede concluir que las medidas sociales promovidos por el Gobierno Revolucionario que asumió el poder el 1ro. de enero de 1959 y el progresivo desarrollo del Sistema Nacional de Salud justifican los positivos resultados alcanzados en el estado de salud de niños, niñas y adolescentes cubanos. A continuación se relacionan los principales eventos favorecedores:

- Los profundos cambios políticos, sociales y económicos que mejoraron el nivel de vida de la población.

- La promulgación de la Ley de Reforma Agraria.

- La promulgación de la Ley de Reforma Educacional, la construcción de 19000 aulas escolares, el 80,0 \% en zonas rurales, la realización de la campaña de alfabetización y el incremento del nivel educacional.

- La creación del servicio médico social, la construcción de hospitales rurales y creación de hogares maternos, los que favorecieron la atención prenatal, al parto, al neonato y al niño.

- Incremento en la formación del personal médico y paramédico (especialización en pediatría, neonatología, ginecoobstetricia, cirugía pediatría y medicina general integral).

- Control de la enfermedad diarreica aguda, el parasitismo intestinal y la desnutrición.

- Creación de consultas de pediatría-puericultura, promoción de la lactancia materna y el programa masivo de inmunizaciones con vacunas contra 13 enfermedades.

- Constitución de los grupos nacionales y provinciales de pediatría y ginecoobstetricia.

- La constitución de los programas de atención integral al niño, de reducción de la mortalidad infantil, de atención materno infantil, del médico y enfermera de la familia, para la reducción del bajo peso al nacer.

- La creación de la Dirección Nacional de Atención Materno Infantil.

- Programa de prevención a los accidentes en el niño. 
- La creación de salas de terapia intensiva pediátricas y neonatales, del cardiocentro pediátrico y de servicios pediátricos de trasplantologia.

- El Programa de detección precoz de anomalías congénitas, fenilcetonuria, anemia drepanocítica, síndrome de Down, hipotiroidismo congénito y errores congénitos del metabolismo.

- Estudio de discapacidades físico-motoras, sensoriales, orgánicas y retraso mental.

\section{REFERENCI AS BI BLI OGRÁFICAS}

1. ONU, UNICEF. Informe sobre el Estado Mundial de la Infancia. New York: UNICEF; 2008.

2. Informe de Salud en Las Américas 2007. Washington: Organización Panamericana de la Salud;2007.

3. Manual de atención integrada a las enfermedades prevalentes de la infancia. Washington: Organización Panamericana de la Salud;2004.

4. Castro Ruz F. La Historia me absolverá. La Habana: Editorial Ciencias Sociales; 1983.

5. Deldado García G. La salud pública en Cuba en el periodo republicano burgués. Conferencia Nueve. Cuad Hist Salud Pública. 1996(81).

6. Cuba y los derechos humanos. Parte III. La Habana: Ministerio de Relaciones Internacionales de la Republica de Cuba; 2006.

7. Constitución de la República de Cuba. Gaceta Oficial de la República de Cuba, edición Extraordinaria número 3 de 31 de enero de 2003.

8. Ley de la salud Pública. Gaceta Oficial de la República de Cuba, edición Ordinaria número 12 del 22 de febrero de 1988.

9. Rojas Ochoa F. Mis Recuerdos del Profesor Lajonchere. Rev Cubana Salud Pública. 2006; 32 (1).

10. Ministerio de Salud Pública de Cuba. Programa Nacional de Inmunización. La Habana: Dirección Nacional de Epidemiología, MINSAP; 1997.

11. Ministerio de Salud Pública de Cuba. Programa de Atención Integral al Niño. La Habana: MINSAP; 1967.

12. Ministerio de Salud Pública de Cuba. Programa de Atención Integral a la Mujer. La Habana: MINSAP; 1967.

13. Ministerio de Salud Pública de Cuba. Programa de Reducción de la Mortalidad Infantil. La Habana: MINSAP; 1970.

14. Ministerio de Salud Pública de Cuba. Programa de Desarrollo de la Especialidad de Pediatría. La Habana: MINSAP; 1987. 
15. Ministerio de Salud Pública de Cuba. Programa de Desarrollo de la Especialidad de Ginecología y Obstetricia. La Habana: MINSAP; 1987.

16. Ministerio de Salud Pública de Cuba. Programa de Desarrollo de la Especialidad de Neonatología. La Habana: MINSAP; 1987.

17. Ministerio de Salud Pública de Cuba. Programa de Desarrollo de la Especialidad de Cirugía Pediátrica. La Habana: MINSAP; 1987.

18. Ministerio de Salud Pública de Cuba. Programa para la Reducción del Bajo Peso al Nacer. La Habana: MINSAP; 1989.

19. Ministerio de Salud Pública de Cuba. Programa del Médico y Enfermera de la Familia. La Habana: MINSAP; 1984.

20. Ministerio de Salud Pública de Cuba. Programa Atención Materno Infantil. La Habana: MINSAP; 1990.

21. Ministerio de Salud Pública de Cuba. Maternidad y Paternidad Responsable. La Habana: MINSAP;2006.

22. Ministerio de Salud Pública de Cuba. Programa "Educa a tu Hijo." La Habana: MI NSAP; 1983.

23. Ministerio de Salud Pública de Cuba. Atención Temprana al Neurodesarrollo. Grupo de Trabajo Dirección Materno Infantil. La Habana: MINSAP; 2006.

24. Ministerio de Salud Pública de Cuba. Informe del Grupo Pediátrico de Hepatotrasplante. Dirección Materno Infantil. La Habana: MINSAP;2006.

25. Valdés Lazo F. Programa Nacional de Prevención de Accidentes en Menores de 20 Años. Dirección Materno Infantil. Ministerio de Salud Pública. La Habana: MINSAP; 2000.

26. Riverón Corteguera R. Estrategias para reducir la mortalidad infantil, en Cuba. 1959-1999. Rev Cubana Pediatria. 2000; 72(3).

27. Ministerio de Salud Pública de Cuba. Anuario Estadístico de Salud. La Habana: MINSAP; 2007.

28. Investigación Perinatal en Cuba 1973. La Habana: Editorial Científico Técnica; 1981.

29. J ordán JR. Desarrollo Humano en Cuba. La Habana: Editorial Científico Técnica; 1979.

30. Estudio Clínico, Psicopedagógico y Social a las Personas con Discapacidad. La Habana: Grupo de Trabajo del MINSAP;2002. 
Recibido: 9 de octubre de 2008.

Aprobado: 18 de octubre de 2008.

María Cecilia Santana Espinosa. Escuela Nacional de Salud Pública. Calle Línea esq. I. El Vedado10400. La Habana, Cuba.

E-mail: cecilia.santana @infomed.sld.cu 